J. Nonlinear Var. Anal. 5 (2021), No. 2, pp. 267-279

Available online at http://jnva.biemdas.com

https://doi.org/10.23952/jnva.5.2021.2.06

\title{
VECTOR PARAMETRIC QUASI-EQUILIBRIUM PROBLEMS FOR THE SUM OF TWO SET-VALUED MAPPINGS
}

\author{
MARZIE DARABI ${ }^{1}$, ZEINAB SOLTANI $^{2, *}$, JAFAR ZAFARANI $^{3}$ \\ ${ }^{1}$ Department of Basic sciences, Golpayegan University of Technology, Isfahan, Iran \\ ${ }^{2}$ Department of Pure Mathematics, Faculty of Mathematical Sciences, \\ University of Kashan, Kashan 87317-53153, Iran \\ ${ }^{3}$ Department of Mathematics, Sheikhbahaee University and University of Isfahan, Isfahan, Iran
}

\begin{abstract}
In this paper, we consider parametric quasi-equilibrium problems for the sum of two setvalued mappings. We present sufficient conditions for the existence of solution of these problems by using a generalized KKM-type theorem in topological vector spaces. Also, we obtain the equivalence between the existence of solution of these problems and scalar equilibrium problem, by a new scalarization.
\end{abstract}

Keywords. Equilibrium problem; Set-valued map; Scalarization; Gap function.

\section{INTRODUCTION AND PRELIMINARIES}

Optimization problems, equilibrium problems and variational inequalities are valuable tools in the modeling of many problems in engineering and economics. For instance, some important models in the study of equilibrium problems, in the engineering sciences (traffic network) and in the economic sciences (oligopolistic market equilibrium problems) have mentioned in [1, $2,3]$. During the last decades, the set-valued optimization theory and its applications have been investigated by many researcher; see, e.g., $[4,5,6,7,8]$ and the references therein. Setvalued optimization is an important and expanding branch of applied mathematics that deals with optimization problems where the objective maps are set-valued maps. Here, we find some efficient elements for the sum of two set-valued maps.

Nonlinear equilibrium problem with constraints can be converted into the constraint optimization problems and variational inequality. In this work, we study also constraint parametric equilibrium problems. Different types of equilibrium problems for set-valued maps in various spaces were intensively studied and many results on the existence of solutions for equilibrium problems were obtained, see $[9,10,11,12,13,14,15,16]$. Moreover, equilibrium problems for the sum of two set-valued mappings were studied in [15, 17]. In fact, Tan and Tinh [17] considered monotonicity assumption, Kassay and Miholca [14] and Kassay, Miholca and Vinh [15] considered C-essential quasimonotonicity assumption for existence of solution of equilibrium

\footnotetext{
${ }^{*}$ Corresponding author.

E-mail addresses: darabi@gut.ac.ir (M. Darabi), z.soltani@kashanu.ac.ir (Z. Soltani), jzaf@zafarani.ir (J. Zafarani).

Received September 26, 2020; Accepted February 27, 2021.
}

(C)2021 Journal of Nonlinear and Variational Analysis 
problems. Here, we focus on set-valued mappings and replace their assumptions with coercivity condition and extend respective results in $[15,17,18]$.

The outline of this paper is as follows: In this section, we define vector parametric quasiequilibrium problems for the sum of two set-valued mappings and some preliminary definitions and results which are utilized in the sequel. In Section 2, we obtain some sufficient conditions for the existence of solution of vector parametric equilibrium problems. In Section 3, the last section, we define gap functions for these problems and obtain the equivalence between existence solution of these problems and their scalar equilibrium problems.

Let $P$ be a Hausdorff topological space, $X$ and $Z$ be Hausdorff topological vector spaces. Let $A$ be nonempty subset of $X$ and $C: X \times P \longrightarrow 2^{Z}$ be a set-valued map such that for all $x \in X$ and for all $p \in P, C(x, p)$ is a closed, convex and pointed cone $(C \cap(-C)=\{0\})$ in $Z$ such that $\operatorname{int} C(x, p) \neq \emptyset$. Suppose that $e: X \times P \longrightarrow Z$ is a continuous vector valued map such that $e(x, p) \in \operatorname{int} C(x, p)$. Also, assume that $K_{1}: A \times P \longrightarrow 2^{A}$ and $K_{2}: A \times P \longrightarrow 2^{A}$ are defined. Let the machinery of the problems be expressed by $F: A \times A \times P \longrightarrow 2^{Z}$ and $G: A \times A \times P \longrightarrow 2^{Z}$. For all subsets $A$ and $B$, we adopt the following notations

$$
\begin{array}{lll}
\beta_{1}(A, B) & \text { means } & A \subseteq B, \\
\beta_{2}(A, B) & \text { means } & A \cap B \neq \emptyset .
\end{array}
$$

For $\beta \in\left\{\beta_{1}, \beta_{2}\right\}$, and $p \in P$, we consider the following vector parametric quasi-equilibrium problem for the sum of two set-valued mappings:

$$
\begin{gathered}
\left(P_{\beta}(p)\right) \text { Find } \bar{x} \in c l K_{1}(\bar{x}, p) \text { such that, } \forall y \in K_{2}(\bar{x}, p), \\
\beta(F(\bar{x}, y, p)+G(\bar{x}, y, p), Z \backslash(-\operatorname{int} C(\bar{x}, p))) .
\end{gathered}
$$

We denote the solution set of Problem $\left(P_{\beta}(p)\right)$ with $S_{\beta}(p)$.

By using the Problem $\left(P_{\beta}(p)\right)$, we can make a mathematical modeling for implicit Cauchy problem (see [19]). In the sequel, we imply special cases of Problem $\left(P_{\beta}(p)\right)$. If $C: X \times P \longrightarrow$ $2^{Z}$ is a fix map, i.e. for any $x \in X$ and $p \in P, C(x, p)=C$ is a convex, closed and pointed cone in $Z$, then

(i) if $C=\mathbb{R}^{+}$, and for each $x \in X$ and $p \in P, K_{1}(x, p)=K_{2}(x, p)$, then Problem $\left(P_{\beta}(p)\right)$ reduces to Problem $(M E P)$ in [19];

(ii) if for all $x \in X$ and $p \in P, K_{1}(x, p)=K_{2}(x, p)$, then Problem $\left(P_{\beta}(p)\right)$ reduces to $(V E P 2)$ and $(V E P 1)$ in [15]. Also, if $F$ and $G$ are single valued then we obtain Problem $(V E P)$ in [20];

(iii) if $\beta=\beta_{2}$ and $F(x, y, p)=<w, y-x>$, such that $T: A \times P \longrightarrow 2^{L(X, Z)}$ and $w \in T(x, p)$, then we obtain parametric generalized vector quasi-variational-like inequality problem in [21];

(iv) if for each $x, y \in A$ and $p \in P$, we define $G(x, y, p)=0$, then

(a) Problem $\left(P_{\beta}(p)\right)$ reduces to vector parametric equilibrium problems, which has been considered in, e.g., [9, 10, 11, 12, 13, 17, 22];

(b) if $F(x, y, p)=H(y, p)-H(x, p)$, that $H: A \times P \longrightarrow 2^{Z}$ then, we have vector parametric optimization problem (see $[16,18,23]$ );

(c) if $F(x, y, p)=<w, y-x>$, such that $T: A \times P \longrightarrow 2^{L(X, Z)}$ and $w \in T(x, p)$ then we obtain vector parametric variational inequality (see $[23,24])$. 
Definition 1.1. A set-valued map $T: X \longrightarrow 2^{Y}$ is said to be:

(1) closed if $\operatorname{Gr}(T)=\{(x, y) \in X \times Y: y \in T(x), x \in X\}$ is a closed subset of $X \times Y$.

(2) upper semi-continuous (u.s.c), iff for each closed set $B \subset Y, T^{-}(B)=\{x \in X: T(x) \cap$ $B \neq \varnothing\}$ is closed in $X$.

(3) lower semi-continuous (1.s.c), iff for each open set $B \subset Y, T^{-}(B)=\{x \in X: T(x) \cap B \neq$ $\varnothing\}$ is open in $X$.

(4) continuous if it is both upper and lower semi-continuous.

(5) intersectionally closed on $A \subseteq X$ if;

$$
\bigcap_{x \in A} \operatorname{cl}(T(x))=\operatorname{cl}\left(\bigcap_{x \in A} T(x)\right) .
$$

(6) topological pseudomonotone, if for all $a, b \in X$,

$$
c l\left(\bigcap_{u \in[a, b]} T(u)\right) \cap[a, b]=\bigcap_{u \in[a, b]} T(u) \cap[a, b] .
$$

(7) KKM map if

$$
\operatorname{conv} A \subseteq \bigcup_{x \in A} T(x), \text { for each } A \in\langle X\rangle,
$$

where, $\langle X\rangle$ denotes the family of all nonempty finite subsets of the set $X$.

The following lemma is a characterization of the upper (resp. lower) semi-continuity of a setvalued mapping in terms of nets (see, for example, [25], Theorems 17.16 and 17.19).

Lemma 1.2. [25] Let $X$ and $Y$ be topological spaces and $T: X \longrightarrow 2^{Y}$ be a set-valued mapping.

(i) If $T$ has compact values, then $T$ is upper semi-continuous iff for every net $x_{\alpha}$ in $X$ converging to $x \in X$ and for any net $y_{\alpha}$ with $y_{\alpha} \in T\left(x_{\alpha}\right)$, there exist $y \in T(x)$ and a subnet $y_{\alpha_{i}}$ of $y_{\alpha}$ converging to $y$.

(ii) $T$ is lower semi-continuous iff for any net $x_{\alpha}$ in $X$ converging to $x \in X$ and each $y \in T(x)$, there exists a net $y_{\alpha}$ converging to $y$, with $y_{\alpha} \in T\left(x_{\alpha}\right)$ for all $\alpha$.

In the following, we introduce the notion of upper and lower mapping corresponding to map $C$ which is a generalization of Definition 2.3 in [15].

Definition 1.3. Let $X$ and $Z$ be Hausdorff topological vector spaces and $A$ be nonempty subset of $X$. Suppose that $C: X \longrightarrow 2^{Z}$ is a set-valued map such that for all $x \in X$ and $C(x)$ is a closed, convex and pointed cone in $Z$ such that $\operatorname{int} C(x) \neq \emptyset$. A set-valued map $F: A \longrightarrow 2^{Z}$ is said to be

(i) upper mapping corresponding to map $C$ if and only if for $i \in\{1,2,3, \ldots, n\}, x_{i} \in A$ and $\lambda_{i} \in[0,1]$ such that $\sum_{i=1}^{n} \lambda_{i}=1$

$$
\sum_{i=1}^{n} \lambda_{i} F\left(x_{i}\right) \subseteq F\left(\sum_{i=1}^{n} \lambda_{i} x_{i}\right)+C\left(\sum_{i=1}^{n} \lambda_{i} x_{i}\right),
$$

(ii) lower mapping corresponding to map $C$ if and only if for $i \in\{1,2,3, \ldots, n\}, x_{i} \in A$ and $\lambda_{i} \in[0,1]$ such that $\sum_{i=1}^{n} \lambda_{i}=1$

$$
F\left(\sum_{i=1}^{n} \lambda_{i} x_{i}\right) \subseteq \sum_{i=1}^{n} \lambda_{i} F\left(x_{i}\right)-C\left(\sum_{i=1}^{n} \lambda_{i} x_{i}\right) .
$$


Example 1. Let $X=\mathbb{R}, Z=\mathbb{R}^{2}, A=[0,1]$ and $C: \mathbb{R} \rightarrow 2^{\mathbb{R}^{2}}$ defined as

$$
C(x)= \begin{cases}\mathbb{R}_{+}^{2}, & x \neq \frac{1}{2}, \\ \{(z, w): z \geq 0,0 \geq w \leq z\}, & x=\frac{1}{2} .\end{cases}
$$

(1) Let $F: A \rightarrow 2^{\mathbb{R}^{2}}$ be a mapping defined by

$$
F(x)= \begin{cases}\{(z, w): 0 \leq z \leq x, w \leq z\}, & x \neq \frac{1}{2}, \\ {[0,1] \times\{0\},} & x=\frac{1}{2} .\end{cases}
$$

It easy to check that $F$ is upper mapping corresponding to map $C$. But $F$ is not lower mapping corresponding to map $C$.

(2) Let $F: A \rightarrow 2^{\mathbb{R}^{2}}$ be a mapping defined by

$$
F(x)= \begin{cases}\{(z, w): 0 \leq z \leq x, w \leq z\}, & x \neq \frac{1}{2}, \\ {\left[\frac{1}{4}, \frac{1}{2}\right] \times\{0\},} & x=\frac{1}{2} .\end{cases}
$$

It easy to check that $F$ is lower mapping corresponding to map $C$. But $F$ is not upper mapping corresponding to map $C$.

The next theorem plays a crucial role in the proof of our main results.

Theorem 1.4. [26] Let $K$ be a nonempty and convex subset of a Hausdorff topological vector space $X$ and $T: K \longrightarrow 2^{K}$. Assume that the following conditions hold:

(i) $T$ is a KKM map;

(ii) for each $A \in\langle K\rangle$, the set-valued map $T \cap \operatorname{conv} A$ is intersectionally closed on convA;

(iii) $T$ is topological pseudomonotone;

(iv) there exist a nonempty subset $B$ of $K$ and a nonempty compact subset $D$ of $K$ such that $\operatorname{conv}(A \cup B)$ is compact, for any $A \in\langle K\rangle$, and for each $y \in K \backslash D$ there exists $x \in$ $\operatorname{conv}(B \cup\{y\})$ such that $y \notin T(x)$.

Then, $\bigcap_{x \in K} T(x) \neq \emptyset$.

\section{Existence of Solution of Parametric Quasi-Equilibrium Problem}

In this section, we obtain some sufficient conditions for the existence of solution of Problem $\left(P_{\beta}(p)\right)$. Motivated by an idea of Chadli at al. [19], let us define $\bar{E}(p)=\left\{x \in A: x \in \operatorname{cl} K_{1}(x, p)\right\}$ and $\Gamma_{\beta}: A \times P \longrightarrow 2^{A}$ such that

$$
\Gamma_{\beta}(y, p)=\{x \in \bar{E}(p): \beta(F(x, y, p)+G(x, y, p), Z \backslash(-\operatorname{int}(C(x, p))))\} .
$$

Now, we define a coercivity condition and obtain sufficient conditions for existence solution of Problem $\left(P_{\beta}(p)\right)$.

Definition 2.1. We say that the pair $\left(\Gamma_{\beta}, K_{1}\right)$ satisfies the coercivity condition (M) if there exist a nonempty subset $B_{0}$ of $X$ and a nonempty compact subset $D_{0}$ of $X$ such that $\operatorname{conv}\left(A_{0} \cup B_{0}\right)$ is compact, for any $A_{0} \in\langle X\rangle$ and for each $y \in X \backslash D_{0}$, there exists $x \in \operatorname{conv}\left(B_{0} \cup\{y\}\right)$ such that if $x \in \bar{E}(p)$ then $y \notin \Gamma_{\beta}(x, p)$, and if $x \notin \bar{E}(p)$, then $y \notin c l K_{1}(x, p)$. 
Theorem 2.2. Let $P$ be a Hausdorff topological space, $X$ and $Z$ be Hausdorff topological vector spaces. Suppose that $A$ is a nonempty convex subset of $X$. Assume that the following conditions hold:

(i) $(F(x, x, p)+G(x, x, p)) \subseteq C(x, p)$, for all $x \in A$;

(ii) $F$ and $G$ are upper mapping corresponding to $C$ in their second argument;

(iii) $W(x, p)=Z \backslash(-\operatorname{int} C(x, p))$ is a closed map;

(iv) the pair $\left(\Gamma_{\beta}, K_{1}\right)$ satisfies the coercivity condition $(\mathrm{M})$.

Therefore,

(a) if $F$ and $G$ are lower semi-continuous in their first argument, then for all $p \in P$, Problem $\left(P_{\beta_{1}}(p)\right)$ has a solution.

(b) if $F$ and $G$ are upper semi-continuous in their first argument and compact valued, then for all $p \in P$, Problem $\left(P_{\beta_{2}}(p)\right)$ has a solution.

(c) if $F$ and $G$ are continuous in their first argument and compact valued, then for all $p \in P$, Problem $\left(P_{\beta}(p)\right)$ has a solution.

Proof. Let $p \in P$. We will show that $\cap_{y \in A} \Gamma_{\beta}(y, p) \neq \emptyset$. First, we prove that the set valued map $\Gamma_{\beta}(., p)$ fulfils the conditions of Theorem 1.4. From (i), we have $y \in \Gamma_{\beta}(y, p) \neq \emptyset$, for all $y \in A$. Hence, we show that $\Gamma_{\beta}$ is KKM map. Suppose on the contrary that there exist $\left\{x_{1}, x_{2}, \ldots, x_{n}\right\}$ and $\lambda_{i} \geq 0$ such that $\sum_{i=1}^{n} \lambda_{i}=1, x=\sum_{i=1}^{n} \lambda_{i} x_{i}$ and $x \notin \cup \cup_{i=1}^{n} \Gamma_{\beta}\left(x_{i}, p\right)$. Then $x \notin \Gamma_{\beta}\left(x_{i}, p\right)$ for all $i \in\{1,2, \ldots, n\}$. Hence, we have

$$
\left(F\left(x, x_{i}, p\right)+G\left(x, x_{i}, p\right)\right) \cap Z \backslash(-\operatorname{int} C(x, p))=\emptyset,
$$

for each $i \in\{1,2, \ldots, n\}$ and

$$
F\left(x, x_{i}, p\right)+G\left(x, x_{i}, p\right) \subseteq(-\operatorname{int} C(x, p)),
$$

and

$$
\sum_{i=1}^{n} \lambda_{i}\left(F\left(x, x_{i}, p\right)+G\left(x, x_{i}, p\right)\right) \subseteq(-\operatorname{int} C(x, p)) .
$$

On the other hand, by (ii),

$$
\begin{aligned}
& \sum_{i=1}^{n} \lambda_{i} F\left(x, x_{i}, p\right) \subseteq F(x, x, p)+C(x, p), \\
& \sum_{i=1}^{n} \lambda_{i} G\left(x, x_{i}, p\right) \subseteq G(x, x, p)+C(x, p) .
\end{aligned}
$$

Then,

$$
\begin{aligned}
\sum_{i=1}^{n} \lambda_{i}\left(F\left(x, x_{i}, p\right)+G\left(x, x_{i}, p\right)\right) & =\Sigma_{i=1}^{n} \lambda_{i} F\left(x, x_{i}, p\right)+\Sigma_{i=1}^{n} \lambda_{i} G\left(x, x_{i}, p\right) \\
& \subseteq F(x, x, p)+C(x, p)+G(x, x, p)+C(x, p) \\
& \subseteq C(x, p)
\end{aligned}
$$

that contradicts (2.3).

Now, we show that, for each $y \in A, \Gamma_{\beta}(y, p)$ is closed. Let $\left(x_{\alpha}\right) \subseteq \Gamma_{\beta}(y, p)$ and $x_{\alpha} \longrightarrow x_{0}$. Since $\left(x_{\alpha}\right) \subseteq \Gamma_{\beta}(y, p)$, then, for all $\alpha$,

$$
\beta\left(F\left(x_{\alpha}, y, p\right)+G\left(x_{\alpha}, y, p\right), Z \backslash\left(-\operatorname{int}\left(C\left(x_{\alpha}, p\right)\right)\right)\right) .
$$

Let $\beta=\beta_{1}$ and condition (a) holds.

Suppose on the contrary that $x_{0} \notin \Gamma_{\beta}(y, p)$. Then $F\left(x_{0}, y, p\right)+G\left(x_{0}, y, p\right) \cap\left(-\operatorname{int}\left(C\left(x_{0}, p\right)\right) \neq\right.$ $\emptyset$, so there exist $w_{0} \in F\left(x_{0}, y, p\right)$ and $z_{0} \in G\left(x_{0}, y, p\right)$ such that $w_{0}+z_{0} \in\left(-\operatorname{int} C\left(x_{0}, p\right)\right)$. By the lower semi-continuity of $F$ and $G$ in their first argument and Lemma 1.2, there exist $w_{\alpha} \in$ 
$F\left(x_{\alpha}, y, p\right)$ and $z_{\alpha} \in G\left(x_{\alpha}, y, p\right)$ such that $w_{\alpha} \longrightarrow w_{0}$ and $z_{\alpha} \longrightarrow z_{0}$. From (2.4), we conclude that $w_{\alpha}+z_{\alpha} \in W\left(x_{\alpha}, p\right)$. But $W$ is a closed map, so $w_{0}+z_{0} \in W\left(x_{0}, p\right)=Z \backslash\left(-\operatorname{int} C\left(x_{0}, p\right)\right)$, then $w_{0}+z_{0} \notin\left(-\operatorname{int} C\left(x_{0}, p\right)\right)$, which is a contradiction. Therefore, $\Gamma_{\beta}(., p)$ is closed.

let $\beta=\beta_{2}$ and condition (b) holds.

Suppose that $x_{0} \notin \Gamma_{\beta}(y, p)$, so $F\left(x_{0}, y, p\right)+G\left(x_{0}, y, p\right) \cap Z \backslash\left(-\operatorname{int}\left(C\left(x_{0}, p\right)\right)=\emptyset\right.$, and

$$
F\left(x_{0}, y, p\right)+G\left(x_{0}, y, p\right) \subset\left(-\operatorname{int}\left(C\left(x_{0}, p\right)\right) .\right.
$$

On the other hand, for all $\alpha$,

$$
F\left(x_{\alpha}, y, p\right)+G\left(x_{\alpha}, y, p\right) \cap Z \backslash\left(-\operatorname{int}\left(C\left(x_{\alpha}, p\right)\right)\right) \neq \emptyset .
$$

Hence, there exist $w_{\alpha} \in F\left(x_{\alpha}, y, p\right)$ and $z_{\alpha} \in G\left(x_{\alpha}, y, p\right)$ such that $w_{\alpha}+z_{\alpha} \in W\left(x_{\alpha}, p\right)$. By the upper semi-continuity of $F$ and $G$ in their first argument and Lemma 1.2, there exist $w_{0} \in$ $F\left(x_{0}, y, p\right), z_{0} \in G\left(x_{0}, y, p\right)$, subnet $w_{\alpha_{i}}$ of $w_{\alpha}$ and subnet $z_{\alpha_{i}}$ of $z_{\alpha}$ such that $w_{\alpha_{i}} \longrightarrow w_{0}$ and $z_{\alpha_{i}} \longrightarrow z_{0}$. But $W$ is a closed map. Hence $w_{0}+z_{0} \in W\left(x_{0}, p\right)=Z \backslash\left(-\operatorname{int} C\left(x_{0}, p\right)\right)$. It follows that $w_{0}+z_{0} \notin\left(-\operatorname{int} C\left(x_{0}, p\right)\right)$, which contradicts (2.5).

If condition (c) holds, then by the above argument, $\Gamma_{\beta}(., p)$ is closed. Therefore, the setvalued map $\Gamma_{\beta}(., p) \cap \operatorname{conv} B$ is intersectionally closed on $\operatorname{conv} B$, for each $B \in\langle A\rangle$.

Furthermore, we show that $\Gamma_{\beta}(., p)$ is a topological pseudomonotone set-valued map. If fix $a, b \in X$. Obviously,

$$
\bigcap_{u \in[a, b]}\left(\Gamma_{\beta}(u, p) \cap[a, b]\right)=\left(\bigcap_{u \in[a, b]} \Gamma_{\beta}(u, p)\right) \cap[a, b] \subseteq c l\left(\bigcap_{u \in[a, b]} \Gamma_{\beta}(u, p)\right) \cap[a, b] .
$$

Conversely; if $x_{0} \in c l\left(\bigcap_{u \in[a, b]} \Gamma_{\beta}(u, p)\right) \cap[a, b]$, then there exists

$$
\left(x_{\alpha}\right) \subseteq\left(\bigcap_{u \in[a, b]} \Gamma_{\beta}(u, p)\right) \cap[a, b]
$$

such that $x_{\alpha} \longrightarrow x_{0}$. So, for each $\alpha, x_{\alpha} \in[a, b]$ and $x_{\alpha} \in \Gamma_{\beta}(u, p)$, for each $u \in[a, b]$. Since $\Gamma_{\beta}(., p)$ is closed valued, then $x_{0} \in \bigcap_{u \in[a, b]}\left(\Gamma_{\beta}(u, p) \cap[a, b]\right)$. Then $\Gamma_{\beta}(., p)$ satisfies the conditions of Theorem 1.4. So, $\cap_{y \in A} \Gamma_{\beta}(y, p) \neq \emptyset$. Therefore, $S_{\beta}(p)$ is nonempty.

Kassay at al. [15], prove the existence of solutions for vector quasi-equilibrium problems for the sum of two set-valued in the setting of topological vector spaces. Their methods are based on a result concerning the existence of maximal elements. In Theorem 2.2, we prove the existence of solutions for vector parametric quasi-equilibrium problem for the sum of two set-valued map with moving cones and with weaker conditions by using generalized KKM-type theorem.

The next example shows that our assumptions in Theorem 2.2 are weaker than those in [15].

Example 2. Let $K_{1}, K_{2}:[0,1] \times[0,1] \longrightarrow 2^{[0,1]}$ and $F, G:[0,1] \times[0,1] \times[0,1] \longrightarrow 2^{\mathbb{R}}$ be defined by $K_{2}(x, p)=[0,1]$,

$$
K_{1}(x, p)= \begin{cases}\left(0, \frac{1}{2}\right], & \text { if } x \in\left[0, \frac{1}{4}\right], p \in Q \\ {[0,1],} & \text { if } x \in\left[\frac{3}{4}, 1\right], p \in Q^{c} \\ \left\{\frac{1}{4}\right\}, & \text { o.w }\end{cases}
$$




$$
F(x, y, p)=G(x, y, p)= \begin{cases}(0,+\infty), & \text { if } x=y, \\ (0,1), & \text { if } x \in\left[0, \frac{1}{4}\right], p \in Q, \\ {[2, \infty),} & \text { if } x \in\left[\frac{3}{4}, 1\right], p \in Q^{c}, \\ (-\infty, 3), & \text { o.w, }\end{cases}
$$

and for all $x, p \in[0,1]$, let $C(x, p)=[0,+\infty)$.

Its easy to see that, conditions (i), (ii) and (iii) of Theorem 2.2 hold. Also, $F$ and $G$ are lower semi-continuous in their first argument. We have

$$
\bar{E}(p)= \begin{cases}{\left[0, \frac{1}{4}\right],} & \text { if } p \in Q, \\ {\left[\frac{3}{4}, 1\right],} & \text { if } p \in Q^{c} .\end{cases}
$$

Moreover, The pair $\left(\Gamma_{\beta_{1}}, K_{1}\right)$ satisfies the coercivity condition (M). Indeed, we set $D_{0}=\left[0, \frac{1}{4}\right] \cup$ $\left[\frac{3}{4}, 1\right]$ and $B_{0}$ any subset of $X$. For any $y \in X \backslash D_{0}=\left(\frac{1}{4}, \frac{3}{4}\right)$, there exists $x=y \in \operatorname{conv}\left(B_{0},\{y\}\right)$ such that for each $p \in P, x \notin \bar{E}(p)$. But $y \notin c l K_{1}(x, p)=\left\{\frac{1}{4}\right\}$ so the pair $\left(\Gamma_{\beta_{1}}, K_{1}\right)$ satisfies the coercivity condition (M). Then all the conditions of Theorem 2.2 hold and

$$
S_{\beta_{1}}(p)= \begin{cases}{\left[0, \frac{1}{4}\right],} & \text { if }, p \in Q, \\ {\left[\frac{3}{4}, 1\right],} & \text { if } p \in Q^{c} .\end{cases}
$$

In the following results, under additional assumptions, we show that $S_{\beta}(p)$ is compact and convex

Theorem 2.3. Let $P$ be a Hausdorff topological space, $X$ and $Z$ be Hausdorff topological vector spaces. Suppose that $A$ is a nonempty subset of a Hausdorff topological vector space $X$. Assume that conditions (i)- (iv) of Theorem 2.2 hold.

Suppose that

(i) $K_{2}$ is lower semi-continuous in its first argument;

(ii) $A$ is a compact set;

Therefore

(a) if $F$ and $G$ are lower semi-continuous, then, for all $p \in P$, solution set $S_{\beta_{1}}(p)$ is compact.

(b) if $F$ and $G$ are upper semi-continuous and compact valued, then, for all $p \in P$, solution set $S_{\beta_{2}}(p)$ is compact.

(c) if $F$ and $G$ are continuous and compact valued, then, for all $p \in P$, solution set $S_{\beta}(p)$ is compact.

Proof. Let $\beta=\beta_{1}$ and $F$ and $G$ satisfy condition (a). Assume that $\left(x_{\alpha}\right)$ is a net in solution set $S_{\beta}(p)$ that $x_{\alpha} \longrightarrow x_{0}$. Suppose that $x_{0} \notin S_{\beta_{1}}(p)$, so there exists $y_{0} \in K_{2}\left(x_{0}, p\right)$ such that

$$
F\left(x_{0}, y_{0}, p\right)+G\left(x_{0}, y_{0}, p\right) \nsubseteq Z \backslash\left(-\operatorname{int} C\left(x_{0}, p\right)\right) .
$$

Thus, there exist $w_{0} \in F\left(x_{0}, y_{0}, p\right)$ and $z_{0} \in G\left(x_{0}, y_{0}, p\right)$ such that $w_{0}+z_{0} \in\left(-\operatorname{int} C\left(x_{0}, p\right)\right)$. Since $K_{2}$ is lower semi-continuous in its first argument, by Lemma 1.2, there exists net $\left(y_{\alpha}\right)$ 
such that $y_{\alpha} \in K_{2}\left(x_{\alpha}, p\right)$ and $y_{\alpha} \longrightarrow y_{0}$. On the other hand, for each $\alpha, x_{\alpha}$ is a solution of Problem $\left(P_{\beta}(p)\right)$, so

$$
F\left(x_{\alpha}, y_{\alpha}, p\right)+G\left(x_{\alpha}, y_{\alpha}, p\right) \subseteq Z \backslash\left(-i n t\left(C\left(x_{\alpha}, p\right)\right)\right)
$$

Also, $F$ and $G$ are lower semi-continuous, by Lemma 1.2, there exist $w_{\alpha} \in F\left(x_{\alpha}, y_{\alpha}, p\right)$ and $z_{\alpha} \in G\left(x_{\alpha}, y_{\alpha}, p\right)$ and $w_{\alpha} \longrightarrow w_{0}$ and $z_{\alpha} \longrightarrow z_{0}$ such that

$$
w_{\alpha}+z_{\alpha} \in Z \backslash\left(-\operatorname{int} C\left(x_{\alpha}, p\right)\right)=W\left(x_{\alpha}, p\right) .
$$

But $W$ is a closed map, so, $w_{0}+z_{0} \in W\left(x_{0}, p\right)$, which is a contradiction.

Let $\beta=\beta_{2}$ and $F$ and $G$ satisfy condition (b).

Suppose that $x_{0} \notin S_{\beta_{2}}(p)$. Then there exists $y_{0} \in K_{2}\left(x_{0}, p\right)$ such that $F\left(x_{0}, y_{0}, p\right)+G\left(x_{0}, y_{0}, p\right) \cap$ $Z \backslash\left(-\operatorname{int} C\left(x_{0}, p\right)\right)=\emptyset$. It follow that

$$
F\left(x_{0}, y_{0}, p\right)+G\left(x_{0}, y_{0}, p\right) \subset\left(-\operatorname{int}\left(C\left(x_{0}, p\right)\right)\right. \text {. }
$$

Since $K_{2}$ is lower semi-continuous in its first argument, by Lemma 1.2, there exists net $\left(y_{\alpha}\right)$ such that, for each $\alpha, y_{\alpha} \in K_{2}\left(x_{\alpha}, p\right)$ and $y_{\alpha} \longrightarrow y_{0}$. On the other hand, for each $\alpha, x_{\alpha}$ is a solution of Problem $\left(P_{\beta}(p)\right)$. Hence,

$$
F\left(x_{\alpha}, y_{\alpha}, p\right)+G\left(x_{\alpha}, y_{\alpha}, p\right) \cap Z \backslash\left(-\operatorname{int}\left(C\left(x_{\alpha}, p\right)\right)\right) \neq \emptyset .
$$

So, there exist $w_{\alpha} \in F\left(x_{\alpha}, y_{\alpha}, p\right)$ and $z_{\alpha} \in G\left(x_{\alpha}, y_{\alpha}, p\right)$ such that $w_{\alpha}+z_{\alpha} \in W\left(x_{\alpha}, p\right)$. By Lemma 1.2, there exist $w_{0} \in F\left(x_{0}, y, p\right), z_{0} \in G\left(x_{0}, y, p\right)$, subnet $\left(w_{\alpha_{i}}\right)$ of $\left(w_{\alpha}\right)$ and subnet $\left(z_{\alpha_{i}}\right)$ of $\left(z_{\alpha}\right)$ such that $w_{\alpha_{i}} \longrightarrow w_{0}$ and $z_{\alpha_{i}} \longrightarrow z_{0}$. But $W$ is a closed map, then $w_{0}+z_{0} \in W\left(x_{0}, p\right)=$ $Z \backslash\left(-\operatorname{int} C\left(x_{0}, p\right)\right)$, and $w_{0}+z_{0} \notin\left(-\operatorname{int} C\left(x_{0}, p\right)\right)$, wich contradicts (2.6).

Suppose that $F$ and $G$ satisfy condition (c). Then, by the above argument, $S_{\beta}(p)$ is a closed subset of $A$. Since $A$ is compact, then $S_{\beta}(p)$ is compact.

Theorem 2.4. Let $P$ be a Hausdorff topological space, $X$ and $Z$ be Hausdorff topological vector spaces. Suppose that $A$ is a nonempty subset of a Hausdorff topological vector space $X$. Assume that conditions of Theorem 2.2 hold.

Suppose that

(i) $F$ and $G$ are lower mapping corresponding to map $(-C)$ in their first argument;

(ii) $W(x, p)=Z \backslash(-\operatorname{int} C(x, p))$ is upper mapping corresponding to map $C$ in its first argument;

Then, for all $p \in P$, solution set $S_{\beta}(p)$ is convex.

Proof. Let $\beta=\beta_{1}$ and $x_{0}, x_{1} \in S_{\beta_{1}}(p)$. So, for each $y_{0} \in K_{2}\left(x_{0}, p\right)$ and $y_{1} \in K_{2}\left(x_{1}, p\right)$,

$$
\begin{aligned}
& F\left(x_{0}, y_{0}, p\right)+G\left(x_{0}, y_{0}, p\right) \subseteq Z \backslash\left(-\operatorname{int} C\left(x_{0}, p\right)\right), \\
& F\left(x_{1}, y_{1}, p\right)+G\left(x_{1}, y_{1}, p\right) \subseteq Z \backslash\left(-\operatorname{int} C\left(x_{1}, p\right)\right) .
\end{aligned}
$$


For all $\lambda \in[0,1]$, since $W$ is upper mapping corresponding to map $C, F$ and $G$ are lower mapping corresponding to map $(-C)$, we have

$$
\begin{array}{rc}
F\left(\lambda x_{0}+(1-\lambda) x_{1}, y, p\right)+G\left(\lambda x_{0}+(1-\lambda) x_{1}, y, p\right) & \subseteq \\
\lambda F\left(x_{0}, y, p\right)+(1-\lambda) F\left(x_{1}, y, p\right)+C\left(\lambda x_{0}+(1-\lambda) x_{1}, p\right) & + \\
\lambda G\left(x_{0}, y, p\right)+(1-\lambda) G\left(x_{1}, y, p\right)+C\left(\lambda x_{0}+(1-\lambda) x_{1}, p\right) & \subseteq \\
\lambda W\left(x_{0}, p\right)+(1-\lambda) W\left(x_{1}, p\right)+C\left(\lambda x_{0}+(1-\lambda) x_{1}, p\right) & \subseteq \\
W\left(\lambda x_{0}+(1-\lambda) x_{1}, p\right)+C\left(\lambda x_{0}+(1-\lambda) x_{1}, p\right) & \subseteq \\
W\left(\lambda x_{0}+(1-\lambda) x_{1}, p\right) . &
\end{array}
$$

Therefore, solution set $S_{\beta_{1}}(p)$ is convex. For $\beta=\beta_{2}$, the proof is similar.

As an immediate consequence of the above results, we obtain the following result.

Corollary 2.5. Let $P$ be a Hausdorff topological space, $X$ and $Z$ be Hausdorff topological vector spaces. Suppose that $A$ is a nonempty, convex and compact subset of $X$. Assume that the following conditions hold:

(1) $(F(x, x, p)+G(x, x, p)) \subseteq C(x, p)$, for all $x \in A$;

(2) $F$ and $G$ are upper mapping corresponding to map $C$ in their second argument;

(3) $F$ and $G$ are lower mapping corresponding to map $(-C)$ in their first argument;

(4) $K_{2}$ is lower semi-continuous in its first argument;

(5) $W(x, p)=Z \backslash(-\operatorname{int} C(x, p))$ is a closed map;

(6) $W(x, p)=Z \backslash(-\operatorname{int} C(x, p))$ is upper mapping corresponding to map $C$ in its first argument

(7) the pair $\left(\Gamma_{\beta}, K_{1}\right)$ satisfies the coercivity condition $(\mathrm{M})$.

Therefore,

(a) if $F$ and $G$ are lower semi-continuous, then for all $p \in P$, solution set $S_{\beta_{1}}(p)$ is nonempty, convex and compact.

(b) if $F$ and $G$ are upper semi-continuous and compact valued, then for all $p \in P$, solution set $S_{\beta_{2}}(p)$ is nonempty, convex and compact.

(c) if $F$ and $G$ are continuous and compact valued, then for all $p \in P$, solution set $S_{\beta}(p)$ is nonempty, convex and compact.

Notice that Example 2 satisfies all the conditions of Corollary 2.5, and, for each $p \in P, S_{\beta_{1}}(p)$ is nonempty, convex and compact.

Let $X$ and $Z$ be a Hausdorff topological vector space, $K$ be a nonempty, closed and convex subset of $X$. Let $\rho \in \mathbb{R}^{+}$and $L(X, Z)$ denote the bounded linear operators space between $X$ and $Z$ for $s \in \operatorname{int} C^{*}$ and $T: X \longrightarrow L(X, Z)$, we define

$$
F(x, y)=\rho \Psi(x, y), G(x, y)=\langle s,\langle T x, y\rangle\rangle,
$$

where $\Psi: K \times K \rightarrow Z$ is a vector-valued bifunctions. Then, we obtain auxiliary vector equilibrium problem in [27]. By the above theorem we can deduce Theorem 4.1 and Theorem 4.2 in [27] under different assumptions.

Here, we can consider the mixed vector equilibrium problem in [27], for all $\varepsilon \in \mathbb{R}^{+}$: $\left(M V E P_{\varepsilon}\right)$ Find $x_{\varepsilon} \in c l K_{1}\left(x_{\varepsilon}, p\right)$ such that $\forall y \in K_{2}\left(x_{\varepsilon}, p\right)$

$$
\beta\left(F\left(x_{\varepsilon}, y, p\right)+\varepsilon G\left(x_{\varepsilon}, y, p\right), Z \backslash\left(-\operatorname{int} C\left(x_{\mathcal{\varepsilon}}, p\right)\right)\right) .
$$


Theorem 2.6. Let $P$ be a Hausdorff topological space, $X$ and $Z$ be Hausdorff topological vector spaces. Suppose that $A$ is a nonempty, convex subset of $X$. Assume that the following conditions hold:

(1) $(F(x, x, p)+G(x, x, p)) \subseteq C(x, p)$, for all $x \in A$;

(2) $F$ and $G$ are upper mapping corresponding to map $C$ in their second argument;

(3) $F$ and $G$ are lower mapping corresponding to map $(-C)$ in their first argument;

(4) $K_{2}$ is lower semi-continuous in its first argument;

(5) $W(x, p)=Z \backslash(-\operatorname{int} C(x, p))$ is a closed map;

(6) $W(x, p)=Z \backslash(-\operatorname{int} C(x, p))$ is upper mapping corresponding to map $C$ in its first argument;

(7) for all $\varepsilon \in \mathbb{R}^{+}, x_{\varepsilon}$ is solution of $\left(M V E P_{\varepsilon}\right)$;

(8) the pair $\left(\Gamma_{\beta}, K_{1}\right)$ satisfies the coercivity condition $(\mathrm{M})$.

Therefore,

(a) if $F$ and $G$ are lower semi-continuous, then for all $p \in P$, any cluster point of $\left\{x_{\varepsilon}\right\}_{\varepsilon>0}$, is a solution of Problem $\left(P_{\beta_{1}}(p)\right)$.

(b) if $F$ and $G$ are upper semi-continuous and compact valued, then for all $p \in P$, any cluster point of $\left\{x_{\varepsilon}\right\}_{\varepsilon>0}$, is a solution of Problem $\left(P_{\beta_{2}}(p)\right)$.

(c) if $F$ and $G$ are continuous and compact valued, then for all $p \in P$, any cluster point of $\left\{x_{\varepsilon}\right\}_{\varepsilon>0}$, is a solution of Problem $\left(P_{\beta}(p)\right)$.

Proof. Let $\beta=\beta_{1}$ and $F, G$ satisfy condition (a). Suppose that $x_{0}$ is a cluster point of $\left\{x_{\varepsilon}\right\}_{\varepsilon>0}$. Then, there exists net $\left\{x_{\varepsilon_{\alpha}}\right\} \subseteq\left\{x_{\varepsilon}\right\}$ such that $x_{\varepsilon_{\alpha}} \longrightarrow x_{0}$. On the other hand, $x_{\varepsilon_{\alpha}}$ is a solution of $\left(M V E P_{\varepsilon_{\alpha}}\right)$. So, for all $y \in K_{2}\left(x_{\varepsilon_{\alpha}}, p\right)$,

$$
F\left(x_{\varepsilon_{\alpha}}, y, p\right)+\varepsilon_{\alpha} G\left(x_{\varepsilon_{\alpha}}, y, p\right) \subseteq Z \backslash-i n t C\left(x_{\varepsilon_{\alpha}}, p\right) .
$$

If $x_{0} \notin S_{\beta_{1}}(p)$, then there exist $y_{0} \in K_{2}\left(x_{0}, p\right), w_{0} \in F\left(x_{0}, y_{0}, p\right)$ and $z_{0} \in G\left(x_{0}, y_{0}, p\right)$ such that $w_{0}+z_{0} \in\left(-\operatorname{int} C\left(x_{0}, p\right)\right)$. But $K_{2}$ is lower semi-continuous in its first argument, by Lemma 1.2, there exists $y_{\varepsilon_{\alpha}} \in K_{2}\left(x_{\varepsilon_{\alpha}}, p\right)$, such that $y_{\varepsilon_{\alpha}} \longrightarrow y_{0}$. Since $F$ and $G$ are lower semi-continuous, from Lemma 1.2, there exist nets $w_{\varepsilon_{\alpha}} \in F\left(x_{\varepsilon_{\alpha}}, y_{\varepsilon_{\alpha}}, p\right)$ and $z_{\varepsilon_{\alpha}} \in G\left(x_{\varepsilon_{\alpha}}, y_{\varepsilon_{\alpha}}, p\right)$ such that $w_{\varepsilon_{\alpha}} \longrightarrow$ $w_{0}$ and $z_{\varepsilon_{\alpha}} \longrightarrow z_{0}$. From (2.9), we have

$$
w_{\varepsilon_{\alpha}}+\varepsilon_{\alpha} z_{\varepsilon_{\alpha}} \in Z \backslash\left(-\operatorname{int} C\left(x_{\varepsilon_{\alpha}}, p\right)\right)=W\left(x_{\varepsilon_{\alpha}}, p\right) .
$$

But $W$ is a closed map. From Lemma 1.2, we have $w_{0}+z_{0} \in Z \backslash\left(-\operatorname{int} C\left(x_{0}, p\right)\right)$, that is a contradiction.

Let $\beta=\beta_{2}$ and $F$ and $G$ satisfy condition (b).

Suppose that $x_{0}$ is a cluster point of $\left\{x_{\varepsilon}\right\}_{\varepsilon>0}$. Then, there exists net $\left\{x_{\varepsilon_{\alpha}}\right\} \subseteq\left\{x_{\varepsilon}\right\}$ such that $x_{\varepsilon_{\alpha}} \longrightarrow x_{0}$. If $x_{0} \notin S_{\beta_{2}}(p)$, then there exists $y_{0} \in K_{2}\left(x_{0}, p\right)$ such that $F\left(x_{0}, y_{0}, p\right)+G\left(x_{0}, y_{0}, p\right) \cap$ $Z \backslash\left(-\operatorname{int} C\left(x_{0}, p\right)\right)=\emptyset$. So

$$
F\left(x_{0}, y_{0}, p\right)+G\left(x_{0}, y_{0}, p\right) \subset\left(-\operatorname{int}\left(C\left(x_{0}, p\right)\right) .\right.
$$

Since $K_{2}$ is lower semi-continuous in its first argument, we conclude from Lemma 1.2 that there exists $y_{\varepsilon_{\alpha}} \in K_{2}\left(x_{\varepsilon_{\alpha}}, p\right)$ such that $y_{\varepsilon_{\alpha}} \longrightarrow y_{0}$. On the other hand, $x_{\varepsilon_{\alpha}}$ is a solution of $\left(M V E P_{\varepsilon_{\alpha}}\right)$. So

$$
F\left(x_{\varepsilon_{\alpha}}, y_{\varepsilon_{\alpha}}, p\right)+\varepsilon_{\alpha} G\left(x_{\varepsilon_{\alpha}}, y_{\varepsilon_{\alpha}}, p\right) \cap Z \backslash\left(-\operatorname{int} C\left(x_{\varepsilon_{\alpha}}, p\right)\right) \neq \emptyset .
$$


Hence, there exist $w_{\varepsilon_{\alpha}} \in F\left(x_{\varepsilon_{\alpha}}, y_{\varepsilon_{\alpha}}, p\right)$ and $z_{\varepsilon_{\alpha}} \in G\left(x_{\varepsilon_{\alpha}}, y_{\varepsilon_{\alpha}}, p\right)$ such that $w_{\varepsilon_{\alpha}}+z_{\varepsilon_{\alpha}} \in W\left(x_{\varepsilon_{\alpha}}, p\right)$. By Lemma 1.2, there exist $w_{0} \in F\left(x_{0}, y, p\right), z_{0} \in G\left(x_{0}, y, p\right)$, subnet $\left(w_{\varepsilon_{\alpha_{i}}}\right)$ of $\left(w_{\varepsilon_{\alpha}}\right)$ and subnet $\left(z_{\alpha_{i}}\right)$ of $\left(z_{\varepsilon_{\alpha}}\right)$ such that $w_{\varepsilon_{\alpha_{i}}} \longrightarrow w_{0}$ and $z_{\varepsilon_{\alpha_{i}}} \longrightarrow z_{0}$. But $W$ is a closed map. So $w_{0}+z_{0} \in$ $W\left(x_{0}, p\right)=Z \backslash\left(-\operatorname{int} C\left(x_{0}, p\right)\right)$, and $w_{0}+z_{0} \notin\left(-\operatorname{int} C\left(x_{0}, p\right)\right)$, which contradicts (2.10).

Suppose that $F$ and $G$ satisfy condition (c). Then, by the above argument, any cluster point of $\left\{x_{\varepsilon}\right\}_{\varepsilon>0}$ is a solution of Problem $\left(P_{\beta}(p)\right)$.

Remark 2.7. Theorem 2.6 is a new version of Theorem 3.1 in [27].

\section{An ScAlarization Method}

In this section, we introduce a new nonlinear scalarization function and define a gap function for Problem $\left(P_{\beta}(p)\right)$. We shall deduce a relation between solution of Problem $\left(P_{\beta}(p)\right)$ and scalar equilibrium problem via its gap function which generalize the results in [20].

Motivated by an idea of Sach [28] and by the Lemma 2 and the Remark 9 in [22], we will define the following notations:

(i) Let $C$ be a convex, closed and pointed cone in $Z$ and $e \in \operatorname{int} C$. If $A$ and $B$ is compact subsets of $X$, then $\varphi_{\beta}: 2^{X} \times 2^{X} \times 2^{Z} \longrightarrow \mathbb{R}$, which is defined by

$$
\varphi_{\beta}(A, B, C):=\min \left\{t \in \mathbb{R}^{+}: \beta(A+B+t e, Z \backslash(-\operatorname{int} C))\right\},
$$

is well defined.

(ii) Let $C: X \times P \longrightarrow 2^{Z}, e(x, p) \in C(x, p)$ and $F, G$ be compact valued. Then, $\psi_{\beta}: X \times X \times Z \longrightarrow$ $\mathbb{R}$, which is defined by

$$
\begin{aligned}
\psi_{\beta}(x, y, p) & :=\varphi_{\beta}(F(x, y, p), G(x, y, p), C(x, p)) \\
& =\min \left\{t \in \mathbb{R}^{+}: \beta(F(x, y, p)+G(x, y, p)\right. \\
& +\operatorname{te}(x, p), Z \backslash(-\operatorname{int} C(x, p)))\}
\end{aligned}
$$

is well defined.

Definition 3.1. A function $L: X \times X \times P \longrightarrow \mathbb{R}^{+}$is said to be a gap function for Problem $\left(P_{\beta}(p)\right)$ corresponding to functions $K_{1}$ and $K_{2}$ iff

(i) $L(x, y, p) \geq 0$, for all $x, y \in X$ and $p \in P$;

(ii) $\bar{x}$ is a solution of Problem $\left(P_{\beta}(p)\right)$ iff $\bar{x} \in c l K_{1}(\bar{x}, p)$ and $L(\bar{x}, y, p)=0$, for all $y \in K_{2}(\bar{x}, p)$.

Lemma 3.2. If $C: X \times P \longrightarrow 2^{Z}$ and $W(x, p)=Z \backslash(-\operatorname{int} C(x, p))$ is a closed map, then the functions $\psi_{\beta}$ is a gap function for Problem $\left(P_{\beta}(p)\right)$, corresponding to functions $K_{1}$ and $K_{2}$.

Proof. The proof with some minor modifications is similar to the proof of Lemma 3 in [22]. Hence, we omit it.

Capata [20] considered a scalar function by assumption $C^{\sharp} \neq \emptyset$. He established a relation between solutions of the strong vector equilibrium problem and the scalarized equilibrium problem. He also showed that the solution set is a nonempty, convex and compact set. In the following theorem, we obtain an equivalence relation between solutions of equilibrium Problem $\left(P_{\beta}(p)\right)$ and the scalar equilibrium problem via its gap function. 
Theorem 3.3. If $\bar{x}$ is a solution of Problem $\left(P_{\beta}(p)\right)$, then, for all $y \in K_{2}(\bar{x}, p)$,

$$
\psi_{\beta}(\bar{x}, y, p)=0 .
$$

Conversely, if, for all $y \in K_{2}(\bar{x}, p), \psi_{\beta}(\bar{x}, y, p)=0$ and $W(x, p)=Z \backslash(-\operatorname{int} C(x, p))$ is closed, then $\bar{x}$ is a solution for Problem $\left(P_{\beta}(p)\right)$.

Proof. It is clear that, if $\bar{x}$ is solution of Problem $\left(P_{\beta}(p)\right)$, then, for all $y \in K_{2}(\bar{x}, p), \psi_{\beta}(\bar{x}, y, p)=$ 0 .

Conversely, if, for all $y \in K_{2}(\bar{x}, p), \psi_{\beta}(\bar{x}, y, p)=0$, then there exists sequence $t_{n} \subseteq \mathbb{R}^{+}$such that $t_{n} \longrightarrow 0$, and, for all $y \in K_{2}(\bar{x}, p)$,

$$
\beta\left(F(\bar{x}, y, p)+G(\bar{x}, y, p)+t_{n} e(\bar{x}, p), Z \backslash(-\operatorname{int} C(\bar{x}, p))\right) .
$$

Suppose that $\bar{x}$ is not a solution of Problem $\left(P_{\beta}(p)\right)$. Then, there exists $y_{0} \in K_{2}(\bar{x}, p) F\left(\bar{x}, y_{0}, p\right)+$ $\left.G\left(\bar{x}, y_{0}, p\right) \subseteq(-\operatorname{int} C(\bar{x}, p))\right)$. So, for each $w \in F(\bar{x}, y, p)$ and $z \in G(\bar{x}, y, p)$, we have $w+z \in$ $(-\operatorname{int} C(\bar{x}, p))$. But $-\operatorname{int} C(\bar{x}, p)$ is an open set and $t_{n} \longrightarrow 0$. Then, there exists $N \in \mathbb{N}$ such that, for all $n \geqslant N, w+z+t_{n} e(\bar{x}, p) \in(-\operatorname{int} C(\bar{x}, p))$, which is a contradiction.

The existence of solutions of Problem $\left(P_{\beta}(p)\right)$ can be investigated via the notions of some approximate solutions. In order to consider this case, we suppose that $\varepsilon \in \mathbb{R}^{+}$, and $p \in P$, we define the set

$$
\begin{aligned}
\Omega_{\beta}(\varepsilon, p)= & \left\{x \in c l K_{1}(x, p):\right. \\
& \left.\beta(F(x, y, p)+G(x, y, p)+\varepsilon e(x, p), Z \backslash(-\operatorname{int} C(x, p)))) \forall y \in K_{2}(x, p)\right\} .
\end{aligned}
$$

Proof of the following results are direct. Hence, we omit their proof.

Proposition 3.4. Let $\varepsilon>0$. Then the following statements hold:

(i) $\cap_{\varepsilon>0} \Omega_{\beta}(\varepsilon, p)=S_{\beta}(p)$;

(ii) $\Omega_{\beta}\left(\varepsilon_{1}, p\right) \subseteq \Omega_{\beta}\left(\varepsilon_{2}, p\right)$, for all $\varepsilon_{1}, \varepsilon_{2}>0$ with $\varepsilon_{1} \leq \varepsilon_{2}$.

Also, we can obtain a nonlinear scalarization of approximate solutions for Problem $\left(P_{\beta}(p)\right)$ by Theorems 3.3 and 3.4 via map $\psi_{\beta}$,

Proposition 3.5. Let $\varepsilon>0$. If $\bar{x} \in \Omega_{\beta}(\varepsilon, p)$, then $\psi_{\beta}(\bar{x}, y, p) \leq \varepsilon$, for all $y \in K_{2}(\bar{x}, p)$. Therefore, $\bar{x} \in \cap_{\varepsilon>0} \Omega_{\beta}(\varepsilon, p)$ iff, $\bar{x} \in S_{\beta}(p)$ iff, $\varphi_{\beta}(\bar{x}, y, p)=0$ and $W(x, p)=Z \backslash(-\operatorname{int} C(x, p))$ is a closed map.

\section{REFERENCES}

[1] C. Baiocchi, A. Capelo, Variational and Quasivariational Inequalities.Applications to Free-Boundary Problems, Wiley, New York, 1984.

[2] D. Kinderlehrer G. Stampacchia, An Introduction to Variational Inequa-lities and their Applications, Academic Press, New York, 1980.

[3] A. Nagurney, Network Economics: A Variational Inequality Approach, Kluwer Academic Publishers, Boston, 1993.

[4] J. P. Aubin, A. Cellina, Differential Inclusions. Set-Valued Maps and Viability Theory, in: Grundlehren der Mathematischen Wissenschaften, vol. 264, Springer-Verlag, Berlin, 1984.

[5] J. Jahn, Vector Optimization Theory, Applications, and Extensions, Springer Verlag, Berlin, 2004.

[6] F.Y. Edgeworth, Mathematical Psychics, Kegan Paul, London, 1881.

[7] E. Klein, A. C. Thompson, Theory of Correspondences. Including Applications to Mathematical Economics, in: Canadian Mathematical Society Series of Monographs and Advanced Texts, New York, 1984. 
[8] V. Pareto, Manuale di Economia Politica (Societa Editrice Libraria, Milano, 1906). English translation: V. Pareto, Manual of Bibliography 467 political economy, translated by A.S. Schwier, Augustus M. Kelley Publishers, New York, 1971.

[9] M. Darabi, J. Zafarani, Tykhonov well-posedness for quasi-equilibrium problems, J. Optim. Theory Appl 165 (2015), 458-479.

[10] Q. H. Ansari, A. P. Farajzadeh, S. Schaible, Existence of solutions of strong vector equilibrium problems, Taiwan. J. Math 16 (2012), 165-178.

[11] M. Castellani, M. Giuli, An existence result for quasiequilibrium problems in separable Banach spaces, J. Math. Anal. Appl 425, (2015) 85-95.

[12] J. W. Chen, Z. Wan, Y. J. Cho, Levitin-Polyak well-posedness by perturbations for systems of set-valued vector quasi-equilibrium problems, Math. Meth Oper. Res 77 (2013), 33-64.

[13] K. Sitthithakerngkiet, S. Plubtieng, Existence theorems of an extension for generalized strong vector quasiequilibrium problems, Fixed Point Theory Appl 2013 (2013), 342.

[14] G. Kassay, M. Miholca, Existence results for vector equilibrium problems given by a sum of two functions, J. Glob. Optim 63 (2015), 195-211.

[15] G. Kassay, M. Miholca, N. T. Vinh, Vector quasi-equilibrium problems for the sum of two multivalued mappings, J. Optim. Theory Appl 169 (2016), 424-442.

[16] A. Dontchev, T. Zolezzi, Well-Posed Optimization Problems, Lecture Notes in Mathematics. Springer, Berlin, 1993.

[17] N. X. Tan, P. N. Tinh, On the existence of equilibrium points of vector functions, Numer. Funct. Anal. and Optim 19 (1998), 141-156.

[18] E. Blum, W. Oettli, From optimization and variational inequalities to equilibrium problems, Math. Stud 63 (1994), 123-145.

[19] O. Chadli, Q. H. Ansari, S. Al-Homidan, Existence of solutions for nonlinear implicit differential equations: An equilibrium problem approach, Numer. Func. Anal. Opt 37 (2016), 1385-1419.

[20] A. Capata, Existence results for globally efficient solutions of vector equilibrium problems via a generalized kkm principle, Acta Math. Sci. 37 (2017), 463-476.

[21] R. P. Agarwal, J. W. Chen, Y. J. Cho, Z. Wan, Stability analysis for parametric generalized vector quasivariational-like inequality problems, J. Inequal. Appl. 2012 (2012), 57.

[22] M. Darabi and J. Zafarani, M-well-posedness and B-well-posedness for vector quasi-equilibrium problems, J. Nonlinear Convex Anal. 17 (2016), 1607-1625.

[23] M. Oveisiha and J. Zafarani, Generalized Minty vector variational-like inequalities and vector optimization problems in Asplund spaces, Optim. Lett. 7 (2013), 709-721.

[24] H. Zhou, Y. Zhou, G. Feng, Iterative methods for solving a class of monotone variational inequality problems with applications, J. Inequal. Appl. 2015 (2015), 68.

[25] C. D. Aliprantis, K. C. Border, Infinite Dimensional Analysis, Springer, Berlin, 2006.

[26] M. Fakhar, M. Lotfipour, J. Zafarani, On the Brezis Nirenberg Stampacchia-type theorems and their applications, J. Global Optim. 55 (2013), 751-770.

[27] O. Chadli, Q. H. Ansari, S. Al-Homidan, Existence of solutions and algorithms for bilevel vector equilibrium problems: An auxiliary principle technique, J. Optim. Theory Appl 172 (2017), 726-758.

[28] P. Sach, New nonlinear scalarization functions and applications, Nonlinear Anal. 75 (2012), 2281-2292. 